\title{
Data Intelligent Low Power High Performance TCAM for IP-Address Lookup Table
}

\author{
K. Mathan1, T. Ravichandran' \\ ${ }^{1}$ Department of Electrical and Electronics Engineering, Hindusthan College of Engineering and Technology, Coimbatore, India \\ ${ }^{2}$ Dean and HoD, SNS College of Tecnology, Coimbatore, India \\ Email: mathan.kaman@gmail.com,dr.t.ravichandran@gmail.com
}

How to cite this paper: Mathan, K. and Ravichandran, T. (2016) Data Intelligent Low Power High Performance TCAM for IP-Address Lookup Table. Circuits and Systems, 7, 3734-3745. http://dx.doi.org/10.4236/cs.2016.711313

Received: March 31, 2016

Accepted: April 15, 2016

Published: September 16, 2016

Copyright $\odot 2016$ by authors and Scientific Research Publishing Inc. This work is licensed under the Creative Commons Attribution International License (CC BY 4.0).

http://creativecommons.org/licenses/by/4.0/ (c) (i) Open Access

\begin{abstract}
This paper represents current research in low-power Very Large Scale Integration (VLSI) domain. Nowadays low power has become more sought research topic in electronic industry. Power dissipation is the most important area while designing the VLSI chip. Today almost all of the high speed switching devices include the Ternary Content Addressable Memory (TCAM) as one of the most important features. When a device consumes less power that becomes reliable and it would work with more efficiency. Complementary Metal Oxide Semiconductor (CMOS) technology is best known for low power consumption devices. This paper aims at designing a router application device which consumes less power and works more efficiently. Various strategies, methodologies and power management techniques for low power circuits and systems are discussed in this research. From this research the challenges could be developed that might be met while designing low power high performance circuit. This work aims at developing Data Aware AND-type match line architecture for TCAM. A TCAM macro of $256 \times 128$ was designed using Cadence Advanced Development Environment (ADE) with $90 \mathrm{~nm}$ technology file from Taiwan Semiconductor Manufacturing Company (TSMC). The result shows that the proposed Data Aware architecture provides around 35\% speed and 45\% power improvement over existing architecture.
\end{abstract}

\section{Keywords}

Low Power, TCAM, Switching Power, Match Line, Searchline, Data Aware and Speech Processing

\section{Introduction}

Power consumption is a major problem while designing VLSI circuit. Few technologies 
were adapted to reduce the power consumption while designing VLSI circuit. One of the technologies is CMOS. Network routers which are used for the packet data transmission, go through the process of IP address matching technique. The matching technique requires the lookup table which has the problem of multiple data access. The new idea of content addressable memory (CAM) is the most popular hardware solution to support the high lookup table speed. The CAM is address by data rather than by address. Since search can be done in parallel, it leads to faster data search than the software lookup. The CAM is widely used in Translation Look aside Buffer (TLB), high associative cache, image processing, database and network routers, etc.; all require fast table lookup.

The routers used in the network applications demand a high speed TCAMs for packet filtering. The TCAM provides the fast look-up table for data and address routing. The network router uses the TCAM and SRAM cell which is used to provide the high speed searching and data storage capacity. However on the fabricating side of TCAM it occupies a large silicon area space. It also creates the problem of stability. Since it occupies a huge area comparatively the power consumption of the TCAM increases drastically. There is a huge power-dissipation problem because of the search operation which is carried out parallel in a chip which is of large size. Just because of the large power dissipation the reliability of the chip is questioned and this again leads to the problem of increase in the package cost of the IC. There were many works reported on the attempt of reducing the power dissipation but that failed to solve the problem of area overhead and noise immunity. Another important problem is the reduction in the yield.

Because of these problems, it attributes to the following shortcomings: 1) increase in chip size, 2) increase in power dissipation, and 3) reduction in the yield, which lead to a production cost overhead. They have to be solved to realize cost-efficient large-scale TCAM chips.

For power consumption, a TCAM consumes power mainly in 3 parts: clock and control, match line, and search line (SL). Thanks to the advancement of match-line design techniques, the power consumption of the former has been greatly reduced. This can be seen from the works of the past three years [1]-[3], where the power consumption for search lines occupies about $54 \%, 71 \%$, and $82 \%$ of the total power, respectively. To tackle the power consumption problem on search lines, pipelining and hierarchical search lines are used in [4]. However, the area and power overhead caused by the pipeline flip-flops and the clock driver diminish the usefulness of the approach.

As discussed in [5] [6], the SLs consume about $16 \%$ - 31\% of total TCAM power. Moreover, after the power reduction in the match-line, the SLs power contribution is increased to $54 \%-82 \%$ to the total power consumption [7]. The power consumption of the SLs can be reduced by several methods, including the use of the hierarchical SL scheme [8] [9], minimizing the switching activity [10], segmenting the SL [7], and recycling the SL charge [11]. However, they all suffer from either performance penalty [7] [8] or complex control circuitry [8] [9] [11]. 
In contrast, this paper introduces a low-power TCAM design that consists of two newly developed schemes, i.e., the refined search enable (RSE) and "don't care" gating (DCG) schemes. Without any performance penalty and complex control circuitry, our design can largely reduce the TCAM power dissipated in the SLs by minimizing both the SL switching activity and the average power consumption per SL switch.

In this paper, we propose a novel Data Aware TCAM (DATCAM) cell which consists of improved Search Enable Line (SEL) and Data Aware Intelligent "Don't Care" Gating (DAIDCG) to solve the problems of power consumptions. The proposed architectures are attractive for realizing small chip size, low-power and high-throughput operation. In addition the architecture has a great impact on the yield improvement. A 4.5-Mb DATCAM design verifies these advances quantitatively.

\section{Proposed Designs}

\subsection{Proposed Data Aware TCAM (DATCAM)}

Data aware proposed TCAM cell is shown in Figure 1, which consists of a data aware block to reduce the redundant computation word bit line WBL and its complement address select line SBL and a discharge transistor N6 [12] [13].

The back to back connected inverted store the value. The simulated lines for different signal are shown in Figure 2. Figure 3 shows the simulated results of the write operation in the TCAM cell. The signal in Figure 2 shows the write bit line (WBL) and its complement (WBLBAR), word line (WL) and select Bit line (SBL). The timing diagram show the storing/write operation being performed for every word line with the select bit line giving the signal for selection.

Figure 3 shows the signals write bit line (WBL), its complement (WBLBAR), word line (WL) and Select Bit line (SBL). The timing diagram show the write "1" operation being carried out in the DATCAM cells.

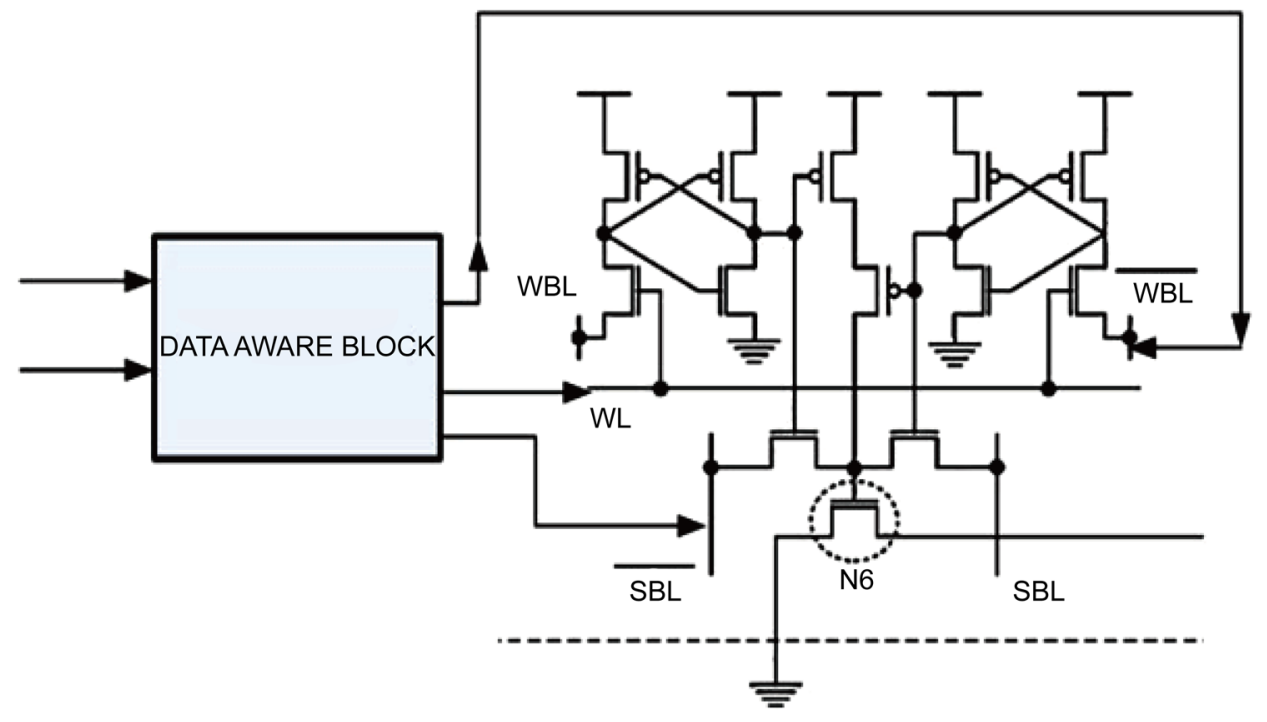

Figure 1. Proposed DATCAM cell. 


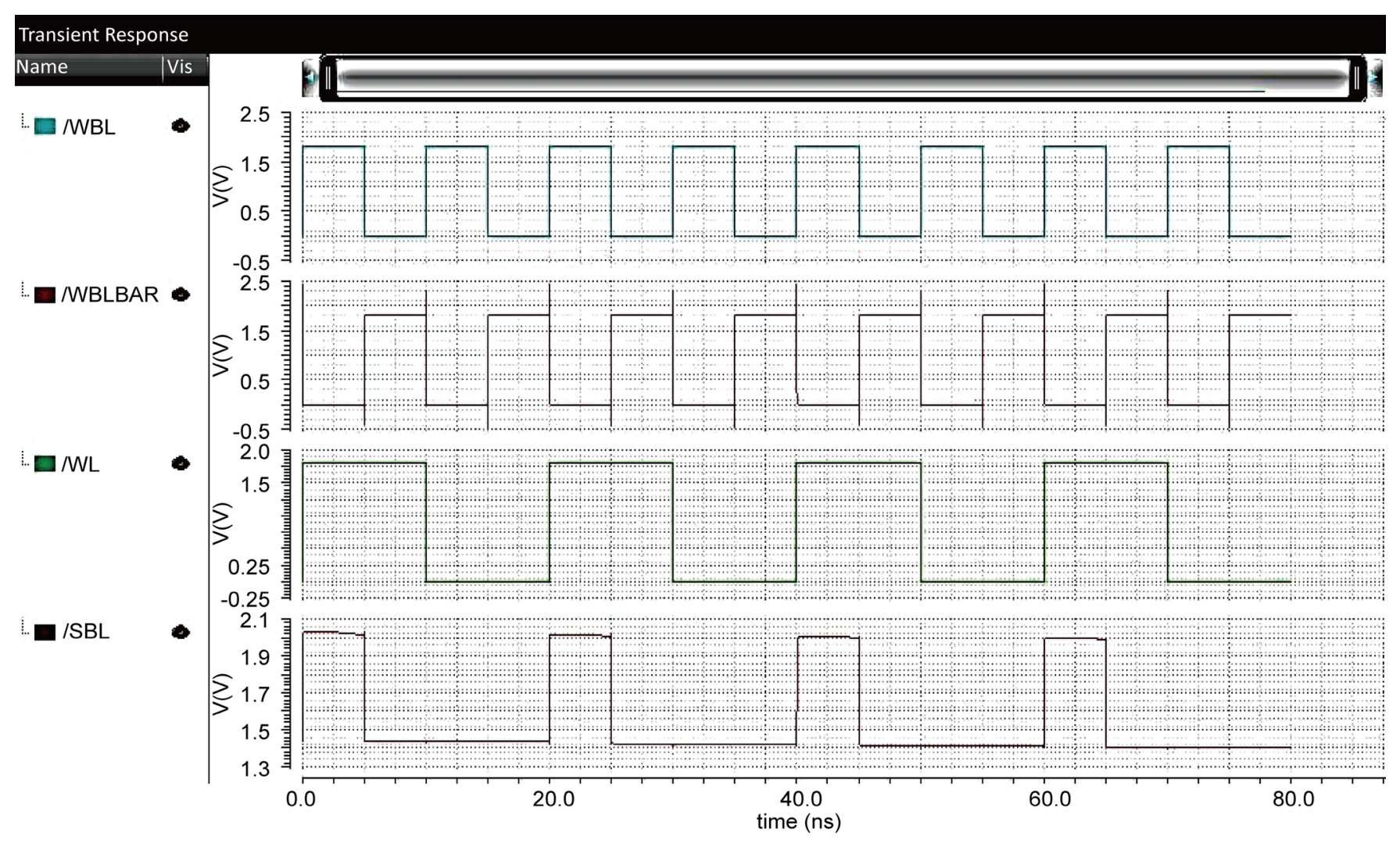

Figure 2. Simulated output of the TCAM cell.

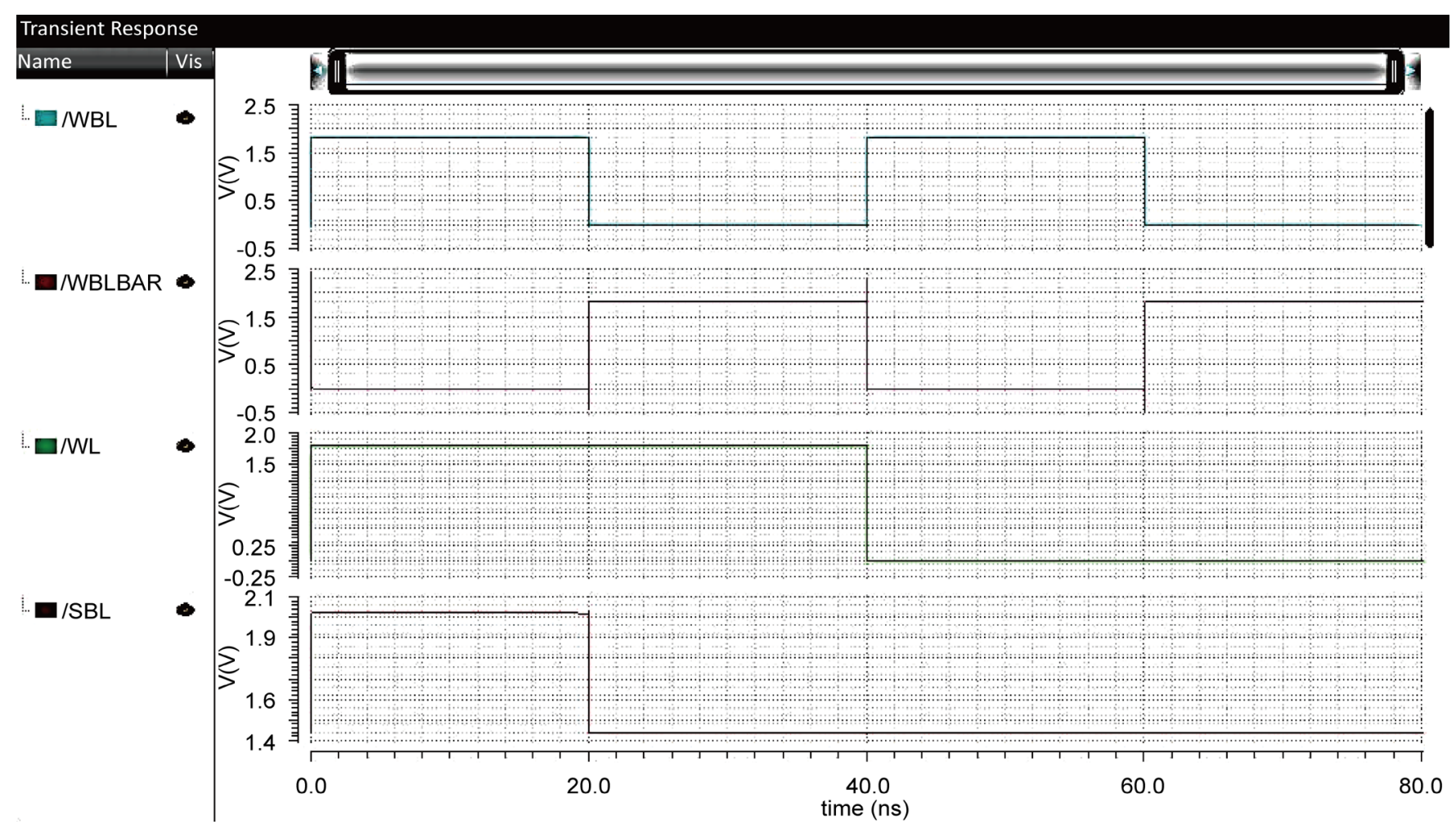

Figure 3. Simulation results of write "1" bit operation performed. 


\subsection{Gating Nodes (GN) Module Design}

In order to gate the search data from being broadcast over the entire SL, our design inserts the GNs to break the entire SL into several segments. As shown in Figure 4, the GN can be easily implemented as a controllable inverter whose function is enabled/ disabled by the corresponding mask bit. For example, if the GN is located on the $\mathrm{i}^{\mathrm{h}}$ cell, the mask value of the $i^{\text {h }}$ cell $M_{i}$ will determine whether the search data can be propagated forward or not. When the th cell is in " $X$ " state, it will cut off both the power and ground sources to deactivate the inverter to further transmit the search data. Otherwise, the inverter will take effect in case of $\mathrm{M}_{\mathrm{i}}=0$. i.e., " 0 " or " 1 " state. The number of cells between two Gns is the definition ot the granularity (G) of DCG scheme. It is critical to optimize both search performance and power saving. If $\mathrm{G}$ is too large, the probability that the first cell of segment is " $\mathrm{X}$ " is low, such that the amount of effective capacitance reduction is insignificant. In contrast, small GN can largely reduce the effective capacitance, but its defect point is the serious propagation delay. In this case, since the number of GNs is too large, the large total inverter delay is detrimental to the data propagation from the first segment to the last segment, such that the degradation in search performance is inevitable. Therefore, an adequate $G$ that benefits both power saving and search performance is very critical to our design. The detailed analysis will be provided in the experimental results. Figure 5 shows the simulated results of the GN module. In Figure 5 the gating operation is done in the read/write operation for the CAM cell the time period of the gating operation is decided by the critical path timing of the DATCAM cell designed which is around 5 ps. The transient response shows the timing diagram of the write operation which is being gate by a clock select signal $s$ and its complement s'.

\subsection{Data Aware Intelligent Don't Care Gating (DAIDCG)}

There are four possible ways of subsequent data transmission, it may have "1" followed by " 0 " or " 1 " similarly " 0 " followed by " 0 " or " 1 " but among the above possibilities the transition which move from "0" to "1" or "1" to "0" will cause the node capacitor to charge and discharge which leads to power consumption. In addition, the Data correlation block will look after the inter signal dependencies that exist between two signals and finally the data computation/comparison is done based on the signal dependencies. In the conventional TCAM with differential SL scheme [14], the ML has to be charged to high during the pre-charge phase, both $S$ and $S$ ' must be discharged to " 0 " to avoid possible short circuit. However, such discharge will increase the unnecessary SL switching activity. It is evident for loss of power. For example, Table 1 shows a search data pattern of five consecutive 0. In case A, i.e. the conventional TCAM design, the gray block contains the values of $S$ which is during the pre-charge phase. Clearly, the number of energy-consuming transitions [15] on SL is 4, but they are all unnecessary switching activities. Consequently, the requirement of discharging both $S$ and $S^{\prime}$ is detrimental to power efficiency. 


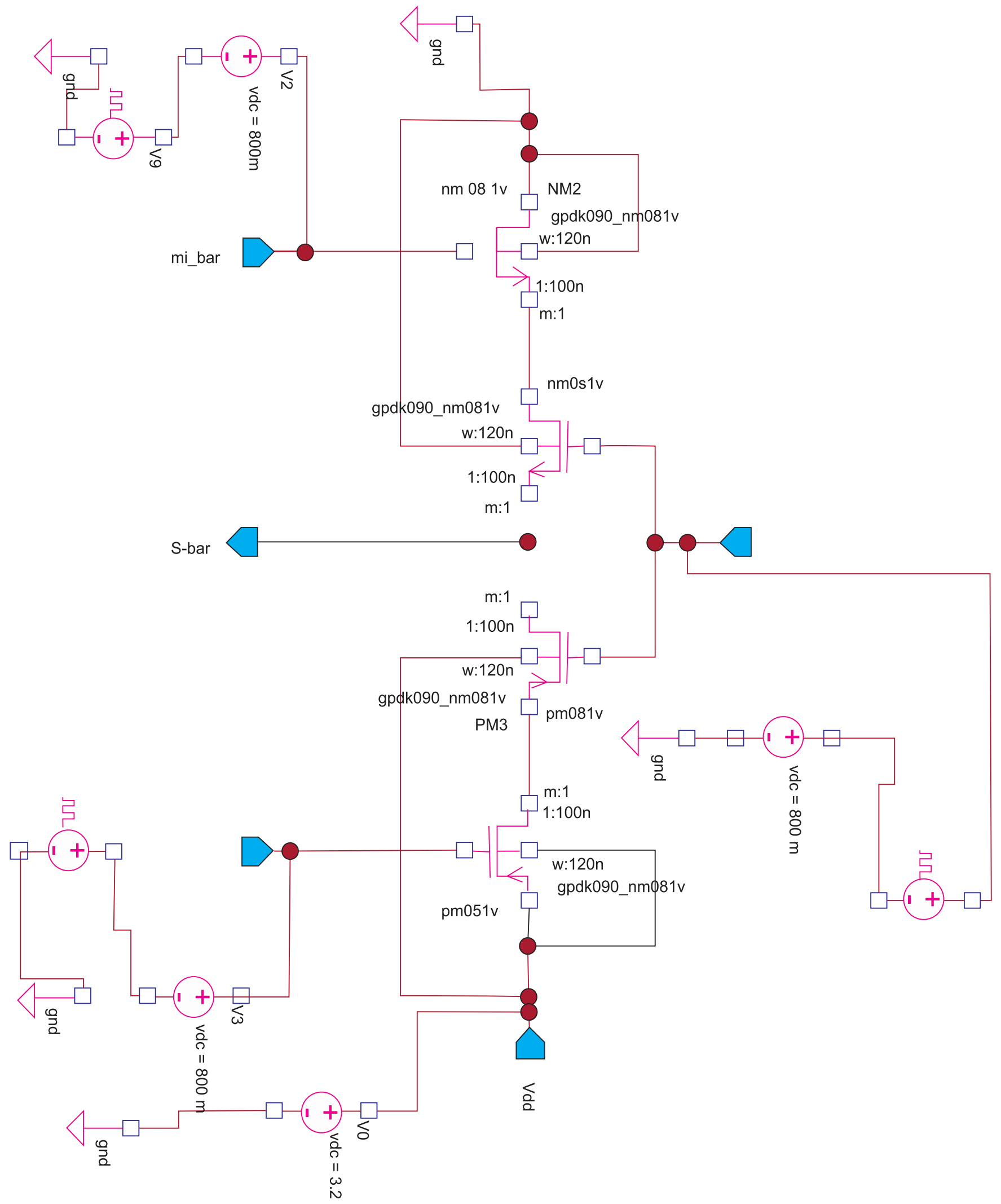

Figure 4. Circuit for GN module. 


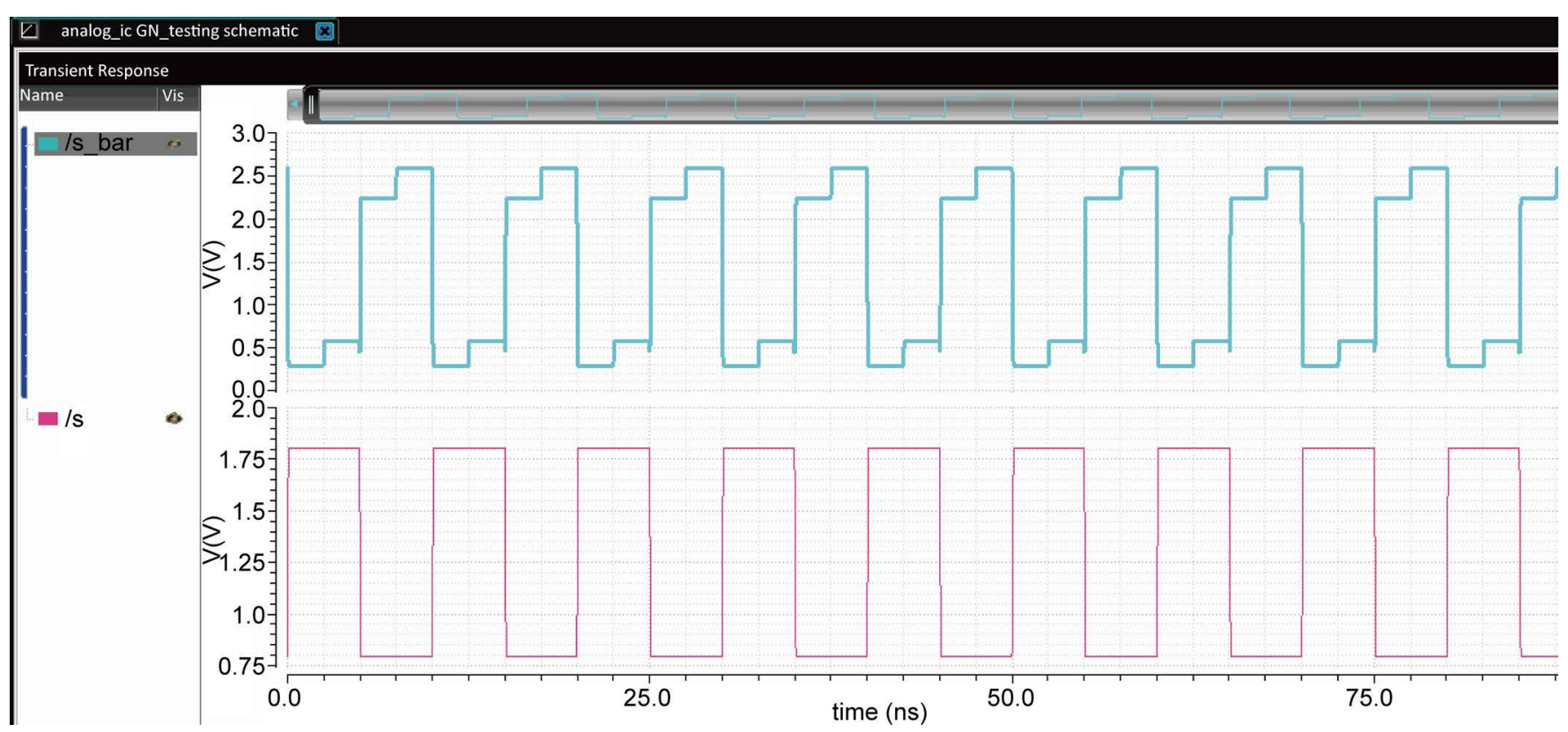

Figure 5. Simulated results of GN output.

Table 1. Search data example of five consecutive 0. Case A/B is the TCAM design without/with SE scheme.

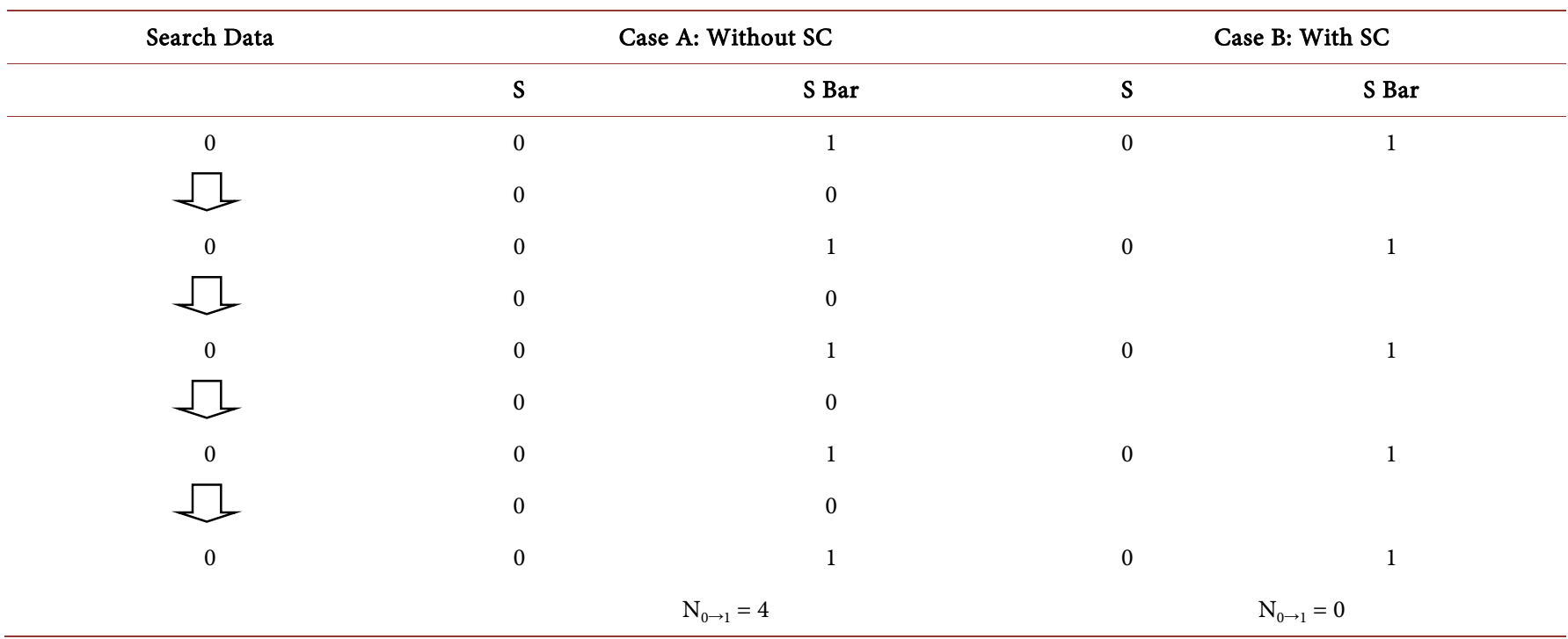

A straightforward solution to this short comings is the introduction of an additional transistor that is used to disconnect the pull-down path during the ML pre-charge, and then enable the search operation during the evaluation phase. This concept is referred to as ISEL scheme, and it is illustrated in Figure 6, from which the additional transistor $\mathrm{N} 3$ is simply controlled by the pre-charge signal, i.e., PRE and ML is shared by all TCAM cells in the same word. During the pre-charge phase, signal line PRE is " 0 " that will pre-charge the ML to "1"; meanwhile, the pull-down path is disconnected by N3. Then, in the evaluation phase, PRE line is set to " 1 " that will turn on N3, so that the ML discharge relies on the match results of the TCAM cells in the board. The effect of the 
SE scheme can be observed from the case B shown in the Table 1. Compared to the conventional TCAM without ISEL scheme [16], the unnecessary transitions are all eliminated.

In the traditional TCAM if the cell is matched in the evaluation phase, then the pull-down path is conducted, whose length is three transistors, i.e., N1, N2, and N3. The number of transistor in the critical path is high for A in the worst case, in which only one TCAM cell is matched, the increased path would lengthen the time to discharge the ML to 0 . Thus, the search performance degradation is inevitable. In order to eliminate the aforementioned performance penalty, the pull-down logic of TCAM cell is refined, as illustrated in Figure 7. During the evaluation phase, i.e., N3 is conducting, there are two possible cases. 1) If the $\mathrm{TCAM}_{0}$ cell is in " $\mathrm{X}$ " state, N4 controlled by $\mathrm{M}$ is turned off to block the transmission of the XOR result. At the same time, $M=1$ will conduct N2 to discharge node $\mathrm{Z}$. Thus, N1 is turned off to disconnect the pull-down path, such that the ML is still high. This is a wild match. 2) In the other case, $M=0$ will

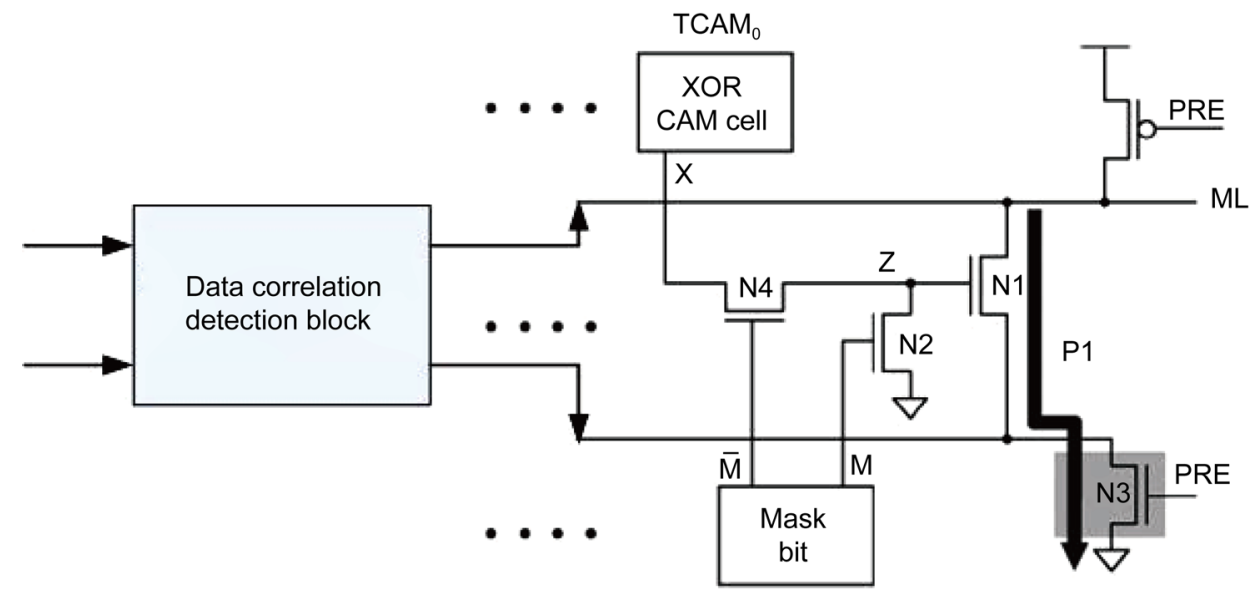

Figure 6. Circuit diagram of search enable line.

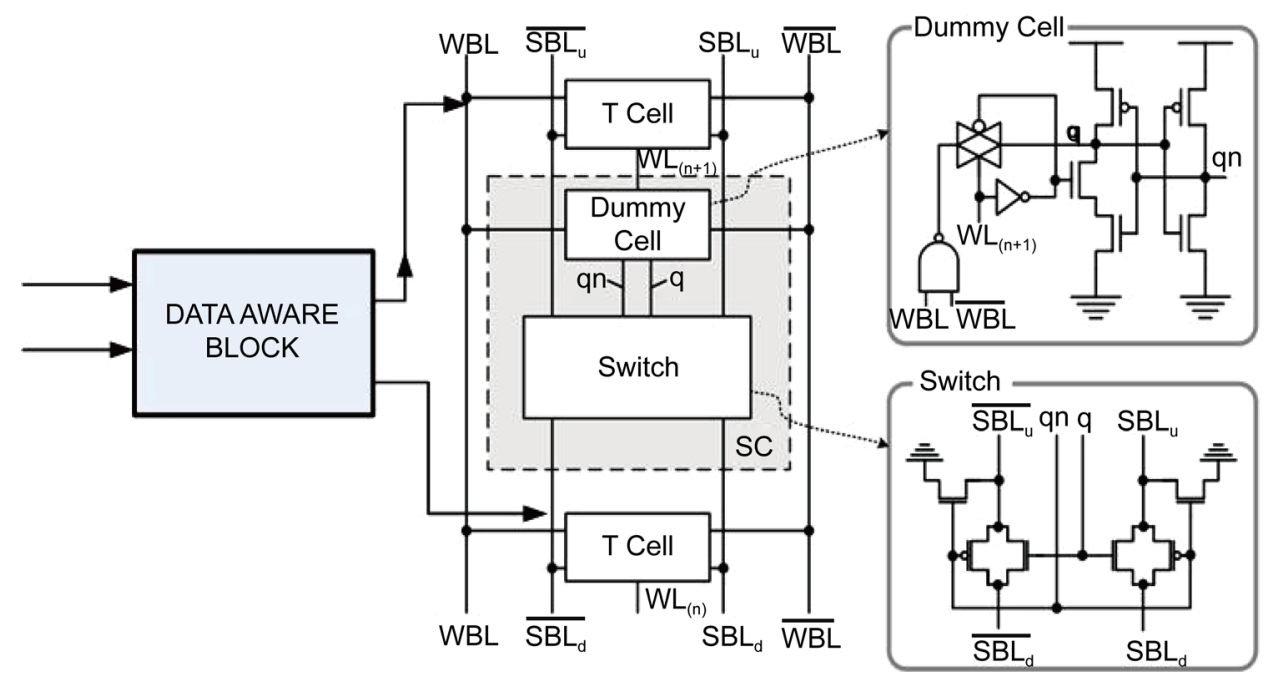

Figure 7. Segmented cell architecture. 
result in such that $\mathrm{N} 2$ is OFF, and N4 is ON. Thus, node $\mathrm{Z}$ follows the XOR result to control N1. The ML is discharged to 0 only when $S$ is not equal to D. Since the length of the conducted pull-down path $(\mathrm{P} 1)$ is 2, the proposed design can achieve the same search performances the conventional TCAM design without the SE scheme. Figure 8 shows the segmented cell simulated output results [17].

\subsection{Segmented Cell Architecture}

In Figure 7 the cells marked as " $x$ " is used to represent the mask (don't care) bits and the example in IP ADDRESS lookup in a progressive pattern. This is used to save the energy because these $\mathrm{x}$ cells need not be used with the search line. But can be used just to pass the signals [18]. This becomes the foundation for the segmented search line design as shown in Figure 7. Energy is saved by writing a " $x$ " in the upper $T$ cell. While doing so the bit and the bit bar are raised high [19]. Out put of the dummy cell q becomes low so that the search path would be cut off and the upper segment of the search line pulled to ground. This inturn would save the energy.

\section{Analysis and Observations}

The circuits are designed with an intention of making the circuit intelligent enough to detect the computation based on the signal correlation and also it tries to reduce the unnecessary switching in the circuits. This subsequently reduces the power consumption of the circuits. The circuits are constructed using virtuoso of cadence and simulated using the spectre. The circuits were simulated using the model file of $90 \mathrm{~nm}$ from

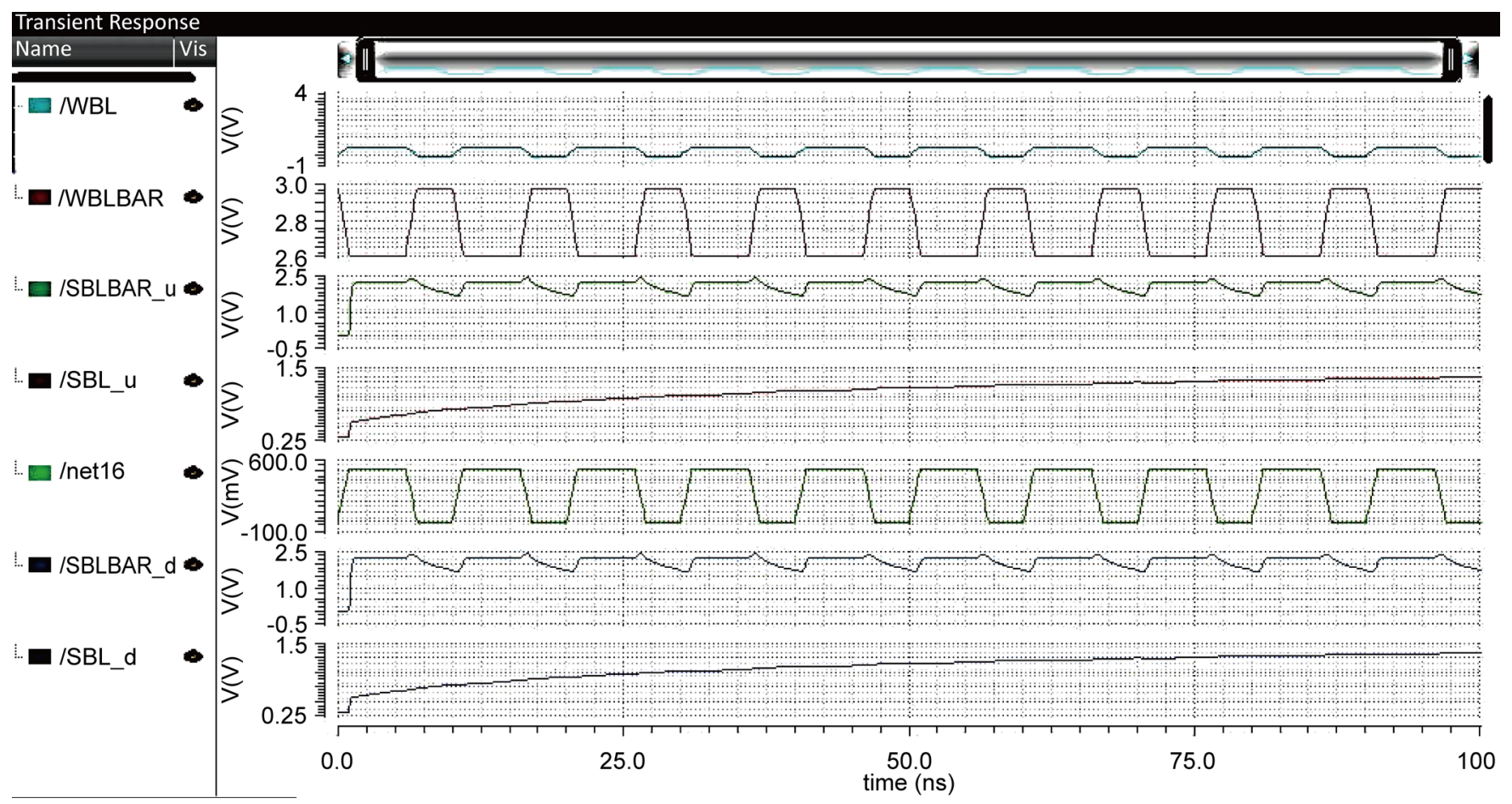

Figure 8. Segmented cell output. 
Table 2. Comparison of the proposed design with the existing works.

\begin{tabular}{ccccc}
\hline Work & Technology $(\mathrm{nm})$ & Voltage (v) & Search time (ns) & Energy (f) /search/bit \\
\hline Jinn Shyan et al. & 130 & 1.2 & 1.10 & 0.35 \\
Igor Arsovski et al. & 130 & 1.2 & 1.40 & 2 \\
Conventional & 180 & 1.8 & 3.04 & 3.7 \\
This work & 90 & 1 & 0.82 & 0.16 \\
\hline
\end{tabular}

Table 3. Energy comparisons with input pattern.

\begin{tabular}{ccc}
\hline & \multicolumn{2}{c}{ Energy } \\
\cline { 2 - 3 } Design & Quite pattern & Switch pattern \\
\cline { 2 - 3 } & $(0->0 / 1->1)$ & $(0->1 / 1->0)$ \\
\hline Conventional & $6.34 \mathrm{E}-05$ & $6.35 \mathrm{E}-05$ \\
This work & $4.05 \mathrm{E}-05$ & $5.20 \mathrm{E}-0.5$ \\
\hline
\end{tabular}

TSMC library. The Verilog simulated in the Modelsim is used in the digital porion of the study design. The results are compared with the previous work in this domain. Table 2 and Table 3 show the comparison of the simulated results.

For accurate evaluation, we use the TSMC 0.18-CMOS technology to layout three IPv4 routing tables, and all data are obtained from the post layout simulation. The table size is fixed at $128 * 32$, i.e., 128 entries by 32 bits, for all tables.

\section{Conclusion}

This paper tries to develop a data aware AND-type match line architecture and complexity less improved search line architecture for TCAM. The proposed architecture in search line tries to explore the feasibility of power reduction in the search line by exploiting the inter- and intra-data dependencies. A TCAM Marco of $256 \times 128 \mathrm{~b}$ was designed using Cadence ADE with $90 \mathrm{~nm}$ technology file from TSMC. The simulated results show that the proposed data aware architecture provides around $40 \%$ reduction in search time and 55\% reduction in the energy consumption over the existing architecture.

\section{References}

[1] Arsovski, I., Chandler, T. and Sheikholeslami, A. (2003) A Ternary Content Addressable Memory (TCAM) Based on $4 \mathrm{~T}$ Static Storage and including a Current-Race Sensing Scheme. IEEE Journal of Solid-State Circuits, 38, 155-158. http://dx.doi.org/10.1109/JSSC.2002.806264

[2] Arsovski, I. and Sheikholeslami, A. (2003) A Mismatch-Dependent Power Allocation Technique for Match-Line Sensing in Content-Addressable Memories. IEEE Journal of SolidState Circuits, 38, 1958-1966. http://dx.doi.org/10.1109/JSSC.2003.818139

[3] Pagiamtzis, K. and Sheikholeslami, A. (2003) Pipelined Match-Lines and Hierarchical Search-Lines for Low-Power Content-Addressable Memories. Proceedings of IEEE Custom 
Integrated Circuits Conference, September 2003, 383-386.

http://dx.doi.org/10.1109/cicc.2003.1249423

[4] Choi, S., Sohn, K., Lee, M.-W., Kim, S., Choi, H.-M., Kim, D., Cho, U.-R., Byun, H.-G., Shin, Y.-S. and Yoo, H.-J. (2004) A 0.7 fJ/Bit/Search, 2.2 ns Search-Time, Hybrid Type TCAM Architecture. IEEE International Solid-State Circuits Conference Digest of Technical Papers, February 2004, 498-499.

[5] Noda, H., Inoue, K., Kuroiwa, M., Igaue, F., Yamamoto, K. and Mattausch, H. (2005) A Cost-Efficient High-Performance Dynamic TCAM with Pipelined Hierarchical Searching and Shift Redundancy Architecture. IEEE Journal of Solid-State Circuits, 40, 245-253. http://dx.doi.org/10.1109/JSSC.2004.838016

[6] Agrawal, B. and Sherwood, T. (2008) Ternary CAM Power and Delay Model: Extensions and Uses. IEEE Transactions on Very Large Scale Integration (VLSI) Systems, 16, 554-564. http://dx.doi.org/10.1109/TVLSI.2008.917538

[7] Wang, J.S., Wang, C.C. and Yeh, C. (2006) TCAM for IP-Address Lookup Using Tree-Style AND-Type Match Lines and Segmented Search Lines. International Solid State Circuits Conference, 577-586.

[8] Huang, P.T., Chang, S.W., Liu, W.Y. and Hwang, W. (2007) A $256 \times 128$ Energy-Efficient TCAM with Novel Low Power Schemes. Proceedings of the International Symposium on VSLI Design, Automation and Test (VLSI-DAT), 1-4. http://dx.doi.org/10.1109/vdat.2007.373204

[9] Yang, B.D. and Kim, L.S. (2005) A Low-Power CAM Using Pulsed NAND-NOR MatchLine and Charge-Recycling SL Driver. IEEE Journal of Solid-State Circuits, 40, 1736-1744. http://dx.doi.org/10.1109/JSSC.2005.852028

[10] Wang, J.-S., et al. (2005) An AND-Type Match-Line Scheme for Energy-Efficient Content Addressable Memories. ISSCC Digest of Technical Papers, February 2005, 464-467.

[11] Arsovski, I., et al. (2003) A Current-Saving Match-Line Sensing Scheme for ContentAddressable Memories. ISSCC Digest of Technical Papers, February 2003, 304-305. http://dx.doi.org/10.1109/isscc.2003.1234309

[12] Noda, H., Inoue, K., Kuroiwa, M., Amo, A., Hachisuka, A., Mattausch, H.J., Koide, K., Soeda, S., Dosaka, K. and Arimoto, K. (2004) A $143 \mathrm{MHz}, 1.1$ W, 4.5 Mb Dynamic TCAM with Hierarchical Searching and Shift Redundancy Architecture. IEEE International Solid-State Circuits Conference Digest of Technical Papers, February 2004, 208-209.

[13] Yamagata, T., Mihara, M., Hamamoto, T., Murai, Y., Kobayashi, T., Yamada, M. and Ozaki, H. (1992) A 288-kb Fully Parallel Content Addressable Memory Using a Stacked-Capacitor Cell Structure. IEEE Journal of Solid-State Circuits, 27, 1927-1933. http://dx.doi.org/10.1109/4.173123

[14] Choi, S., et al. (2004) A 0.7 fJ/Bit/Search, 2.2 ns Search Time, Hybrid Type TCAM Architecture. ISSCC Digest of Technical Papers, February 2004, 498-507.

[15] National Chung Hsing University, Taichung [Online]. http://www.nchu.edu.tw

[16] Shah, D. and Gupta, P. (2001) Fast Updating Algorithms for TCAMs. IEEE Micro, 21, 3647. http://dx.doi.org/10.1109/40.903060

[17] Lin, C.S., Chang, J.C. and Liu, B.D. (2003) A Low-Power Precomputation-Based Fully Parallel Content Addressable Memory. IEEE Journal of Solid-State Circuits, 38, 654-662. http://dx.doi.org/10.1109/JSSC.2003.809515

[18] Akhbarizadeh, M.J., Nourani, M., Vijayasarathi, D.S. and Balsara, P.T. (2006) A Nonredundant Ternary CAM Circuit for Network Search Engines. IEEE Transactions on Very Large Scale Integration (VLSI) Systems, 14, 268-278. 
http://dx.doi.org/10.1109/TVLSI.2006.871760

[19] Baeg, S. (2008) Low Power Ternary Content-Addressable Memory Design Using a Segmented Match Line. IEEE Transactions on Circuits and Systems I: Regular Papers, 55, 1485-1494. http://dx.doi.org/10.1109/TCSI.2008.916624

Submit or recommend next manuscript to SCIRP and we will provide best service for you:

Accepting pre-submission inquiries through Email, Facebook, LinkedIn, Twitter, etc. A wide selection of journals (inclusive of 9 subjects, more than 200 journals)

Providing 24-hour high-quality service

User-friendly online submission system

Fair and swift peer-review system

Efficient typesetting and proofreading procedure

Display of the result of downloads and visits, as well as the number of cited articles

Maximum dissemination of your research work

Submit your manuscript at: http://papersubmission.scirp.org/ 